\title{
Orbital Granuloma Formation Following Autoinjection of Paraffin Oil: Management Considerations
}

\author{
Simi D. Cadmus, MD; Lindsay L. Thelin, MD; Ivan Vrcek, MD
}

\section{PRACTICE POINTS}

- The initial presentation of a foreign-body granulomatous process in a patient with surreptitious use of nonmedical filler can mimic infection; thus, careful history and diagnostic measures are paramount.

- Treatment of paraffin oil granuloma can be multifactorial and involves supportive care, systemic antiinflammatory medications, time, and surgery.

- When a paraffin granuloma involves the orbital region, particular care is required to avoid long-term complications including cicatricial lagophthalmos, ectropion, or retractions, which can be mitigated with the help of oculoplastic surgery.

To the Editor:

Injectable fillers are an increasingly common means of achieving minimally invasive facial rejuvenation. In the hands of well-trained practitioners, these compounds typically are well tolerated, effective, and have a strong safety profile ${ }^{1}$; however, there have been reports of complications, including vision loss, ${ }^{2}$ orbital infarction, ${ }^{3}$ persistent inflammatory nodules, ${ }^{4}$ and infection. ${ }^{4,5}$ Paraffin, a derivative of mineral oil, currently is used in cosmetic products and medical ointments. ${ }^{6}$ In the early 1900s, it often was injected into the body for various medical procedures, such as to create prosthetic testicles, to treat bladder incontinence, and eventually to correct facial contour defects. ${ }^{78}$ Due to adverse effects, injection of paraffin oil was discontinued in the Western medical community around the time of World War I. ${ }^{7}$ Unfortunately, some patients continue to self-inject paraffin oil for cosmetic purposes today. We present a case of foreign-body granuloma formation mimicking periorbital cellulitis following self-injection of paraffin oil. Our patient developed serious periorbital sequelae that required surgical intervention to restore normal anatomic function.

A 60-year-old woman who was otherwise healthy presented to the emergency department with facial swelling and a rash of 2 weeks' duration. She reported that she had purchased what she believed was a cosmetic product at a local flea market 2 weeks prior to presentation. Her purchase included needles and a syringe with verbal instructions for injection into the face. She was told the product was used to treat wrinkles and referred to the injectable material as "oil" when providing her history. She reported that she had injected the material into the bilateral lower eyelids, left lateral lip, and left lateral chin. Three days later, she developed tingling and itching with swelling and redness at the injection sites. The patient was evaluated by the emergency department team and was prescribed a 10-day course of clindamycin empirically for suspected facial cellulitis.

The patient returned to the emergency department 12 days later upon completion of the antibiotic course with worsening edema and erythema. Examination revealed indurated, erythematous, and edematous warm plaques on the face that were concentrated around the prior injection sites with substantial periorbital erythema and edema (Figure 1). A consultation with oculoplastic

Dr. Cadmus is from the Division of Dermatology, University of Texas Dell Medical School, Austin. Dr. Thelin is from the Department of Dermatology, Confluence Health, Wenatchee, Washington. Dr. Vrcek is from Texas Eye Plastics, Dallas.

The authors report no conflict of interest.

Correspondence: Simi D. Cadmus, MD, 1701 Trinity St, Ste 7.802, Austin, TX78712 (simi.cadmus@gmail.com). doi: 10.12788 /cutis. 0117 
FIGURE 1. A, Multiple erythematous firm plaques on the face. $B$, Excessive erythema, induration, and swelling of the right periorbital skin.
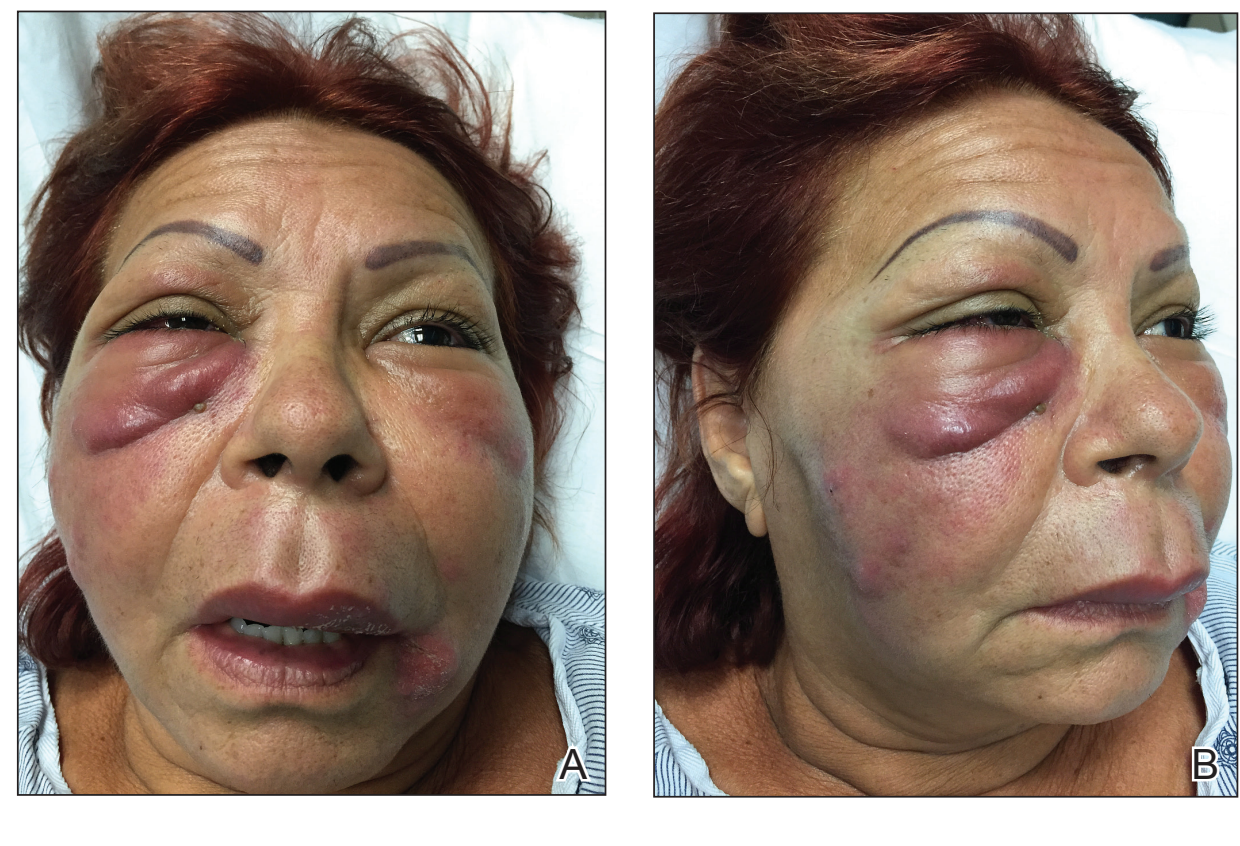

surgery was obtained. Mechanical ptosis of the right eyelid was noted. Visual acuity was 20/30 in both eyes with habitual correction. Intraocular pressure was soft to palpation, and the pupils were round and reactive with no evidence of a relative afferent pupillary defect. Extraocular motility was intact bilaterally. Examination of the conjunctiva and sclera revealed bilateral conjunctival injection with chemosis of the right eye. The remainder of the anterior and posterior segment examination was within normal limits bilaterally.

Computed tomography of the face showed extensive facial and periorbital swelling without abscess. A dermatology consultation was obtained. Two $4-\mathrm{mm}$ punch biopsies were obtained from the left lower face and were sent for hematoxylin and eosin stain and tissue culture (bacterial, fungal, and acid-fast bacillus). Given the possibility of facial and periorbital cellulitis, empiric intravenous antibiotic therapy was initiated.

The tissue culture revealed normal skin flora. The biopsy results indicated a foreign-body reaction consistent with paraffin granuloma (Figures 2 and 3). Fite-Faraco, Grocott-Gomori methenamine-silver, and periodic acidSchiff stains were all negative for infection. A diagnosis of foreign-body granuloma was established. Oral minocycline at a dosage of $100 \mathrm{mg}$ twice daily was started, and the patient was discharged.

After 4 weeks of minocycline therapy, the patient showed no improvement and returned to the emergency department with worsening symptoms. She was readmitted and started on intravenous prednisone $(1.5 \mathrm{mg} / \mathrm{kg} / \mathrm{d})$. Over the ensuing 5 days, the edema, erythema, conjunctival injection, and chemosis demonstrated notable improvement. She was subsequently discharged on an oral prednisone taper. Unfortunately, she did not respond to a trial of intralesional

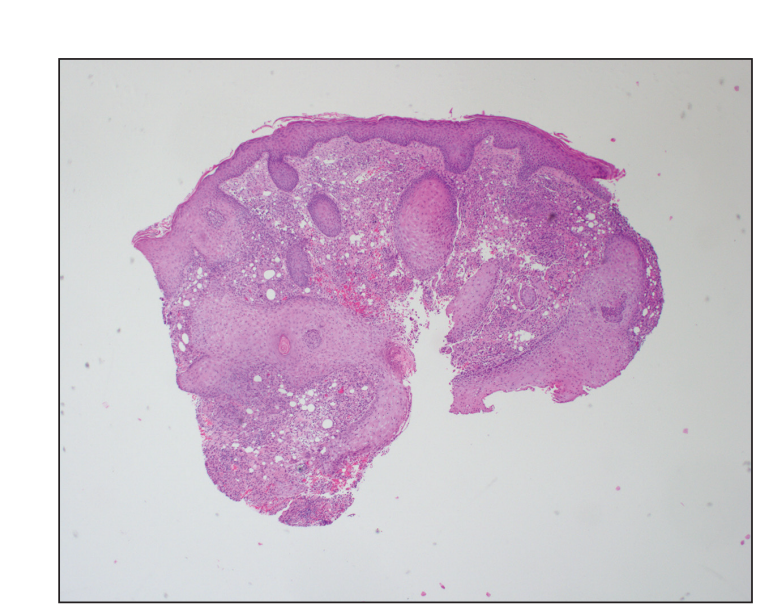

FIGURE 2. Pseudoepitheliomatous hyperplasia with a mixed dermal infiltrate and round cystic spaces in the dermis (H\&E, original magnification $\times 4)$.

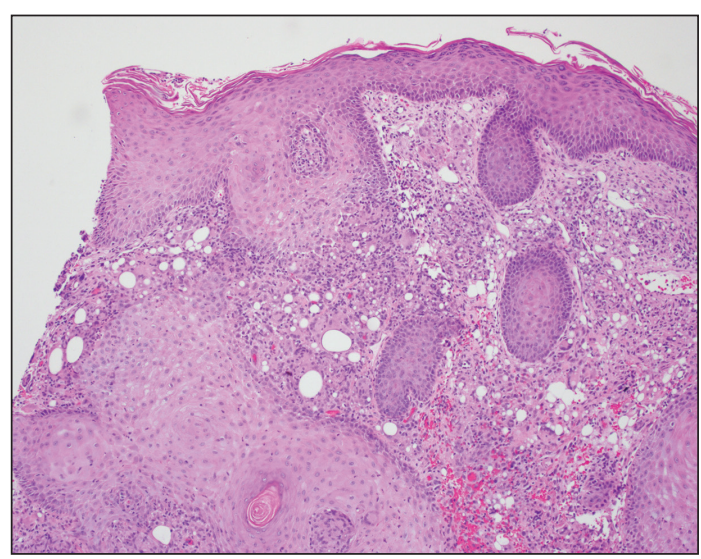

FIGURE 3. Mixed inflammation of the skin including foreign body-type giant cells admixed with the cystic dermal spaces (H\&E, original magnification $\times 10)$. 
steroid injections to an area of granuloma formation on the left chin performed in the hospital before she was discharged.

In the ensuing months, she began to develop cicatricial ectropion of the right lower eyelid and mechanical ptosis of the right upper eyelid. Ten months after initial self-injection, staged surgical excision was initiated by an oculoplastic surgeon (I.V.) with the goal of debulking the periorbital region to correct the ectropion and mechanical ptosis. A transconjunctival approach was used to carefully excise the material while still maintaining the architecture of the lower eyelid. The ectropion was surgically corrected concurrently.

One month after excision, serial injections of 5-fluorouracil (5-FU) and triamcinolone acetonide $40 \mathrm{mg} / \mathrm{mL}$ were administered to the right lower eyelid and anterior orbit for 3 months. Fifteen weeks after the first surgery, a second surgery was performed to address residual medial right lower eyelid induration, right upper eyelid mechanical ptosis, and left orbital inflammation. During the postoperative period, serial monthly injections of 5-FU and triamcinolone acetonide were again performed beginning at the first postoperative month.

The surgical excisions resulted in notable improvement 3 months following excision (Figure 4). The patient noted improved ocular surface comfort with decreased foreign-body sensation and tearing. She also was pleased with the improved cosmetic outcome.

Crude substances such as paraffin, petroleum jelly, and lanolin were used for aesthetic purposes in the late 19th and early 20th centuries, initially with satisfying results; however, long-term adverse effects such as hardening of the skin, swelling, granuloma formation, ulceration, infections, and abscesses have discouraged its use by medical professionals today. ${ }^{5}$ Since paraffin is resistant to degradation and absorption, foreign-body reactions may occur upon injection. These reactions are characterized by replacement of normal subcutaneous tissue by cystic spaces of paraffin oil and/or calcification, similar to the appearance of Swiss cheese on histology and surrounded by various inflammatory cells and fibrous tissue., ${ }^{9,10}$

Clinically, there is an acute inflammatory phase followed by a latent phase of chronic granulomatous inflammation that can last for years. ${ }^{10}$ Our patient presented during the acute phase, with erythematous and edematous warm plaques around the eye mimicking an orbital infection.

The treatment of choice for paraffin granuloma is complete surgical excision to prevent recurrence. ${ }^{6,9}$ However, intralesional corticosteroids are preferred in the facial area, especially if complete removal is not possible. ${ }^{10}$ Intralesional corticosteroid injections inhibit fibroblast and macrophage activity as well as the deposition of collagen, leading to reduced pain and swelling in most cases. ${ }^{11}$ Additionally, combining antimitotic agents such as 5-FU with a corticosteroid might reduce the risk for cortisone skin atrophy. ${ }^{12}$ In our case, the patient did not respond to combined 5-FU with intralesional

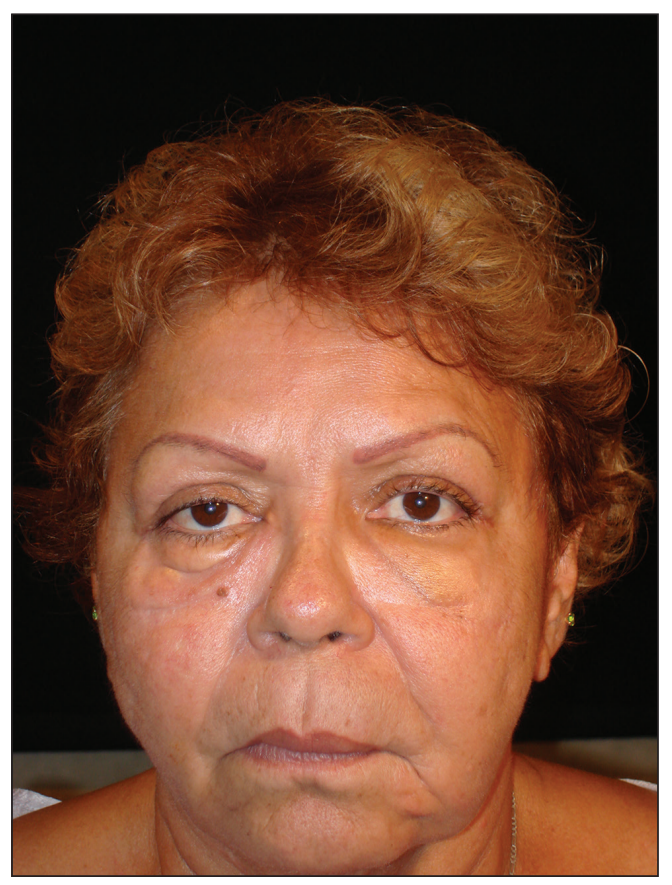

FIGURE 4. Clinical improvement of the plaques and swelling was noted 3 months following excision.

steroids and required oral corticosteroids while awaiting serial excisions.

Our case highlights several important points in the management of paraffin granuloma. First, the clinician must perform a thorough patient history, as surreptitious use of non-medical-grade fillers is more common than one might think..$^{13}$ Second, the initial presentation of these patients can mimic an infectious process. Careful history, testing, and observation can aid in making the appropriate diagnosis. Finally, treatment of these patients is complex. The mainstays of therapy are systemic anti-inflammatory medications, time, and supportive care. In some cases, surgery may be required. When processes such as paraffin granulomas involve the periorbital region, particular care is required to avoid cicatricial lagophthalmos, ectropion, or retraction. Thoughtful surgical manipulation is required to avoid these complications, which indeed may occur even with the most appropriate interventions.

\section{REFERENCES}

1. Duker D, Erdmann R, Hartmann V, et al. The impact of adverse reactions to injectable filler substances on quality of life: results from the Berlin Injectable Filler Safety (IFS) — study. J Eur Acad Dermatol Venereol. 2016;30:1013-1020.

2. Prado G, Rodriguez-Feliz J. Ocular pain and impending blindness during facial cosmetic injections: is your office prepared? [published online December 28, 2016]. Aesthetic Plast Surg. 2017;41:199-203.

3. Roberts SA, Arthurs BP. Severe visual loss and orbital infarction following periorbital aesthetic poly-(L)-lactic acid (PLLA) injection. Ophthalmic Plast Reconstr Surg. 2012;28:E68-E70.

4. Cassuto D, Pignatti M, Pacchioni L, et al. Management of complications caused by permanent fillers in the face: a treatment algorithm. Plast Reconstr Surg. 2016;138:215E-227E. 
5. Haneke E. Adverse effects of fillers and their histopathology. Facial Plast Surg. 2014;30:599-614.

6. Friedrich RE, Zustin J. Paraffinoma of lips and oral mucosa: case report and brief review of literature. GMS Interdiscip Plast Reconstr Surg DGPW. 2014;3:Doc05.

7. Matton G, Anseeuw A, De Keyser F. The history of injectable biomaterials and the biology of collagen. Aesthetic Plast Surg. 1985; 9:133-140.

8. Glicenstein J. Les premiers fillers, Vaseline et paraffine. du miracle a la catastrope. Ann Chir Plast Esthet. 2007;52:157-161.

9. Cohen JL, Keoleian CM, Krull EA. Penile paraffinoma: self-injection with mineral oil. J Am Acad Dermatol 2002;47:S251-S253.
10. Legaspi-Vicerra ME, Field LM. Paraffin granulomata, "witch's chin," and nasal deformities excision and reconstruction with reduction chinplasty and open rhinotomy resection. J Clin Aesthet Dermatol 2010;3:54-58.

11. Carlos-Fabuel L, Marzal-Gamarra C, Marti-Alamo S, et al. Foreign body granulomatous reactions to cosmetic fillers. J Clin Exp Dent. 2012;4:E244-E247.

12. Lemperle G, Gauthier-Hazan N. Foreign body granulomas after all injectable dermal fillers: part 2. treatment options. Plast Reconstr Surg. 2009;123:1864-1873.

13. Seok J, Hong JY, Park KY, et al. Delayed immunologic complications due to injectable fillers by unlicensed practitioners: our experiences and a review of the literature. Dermatol Ther. 2016;29:41-44. 